\title{
Peculiarities of the temperature dependences of silicon solar cells illuminated with light simulator
}

\author{
A.V. Sachenko, V.P. Kostylyov, R.M. Korkishko, M.R. Kulish, I.O. Sokolovskyi, V.M. Vlasiuk, \\ D.V. Khomenko \\ V. Lashkaryov Institute of Semiconductor Physics, NAS of Ukraine, \\ 45, prospect Nauky, 03028 Kyiv, Ukraine, \\ E-mail:sach@isp.kiev.ua
}

\begin{abstract}
Characteristics of basic silicon solar cells are experimentally researched and theoretically modeled using photons of incandescent lamps as sunlight simulator. It was established that increasing temperature evokes significant acceleration of short-circuit current growth. The reason of it is the shift of simulator spectrum to the higher wavelengths region as compared to the Sun one. This effect leads to a reduction in efficiency decrease for simulated sunlight with the increase of temperature. It should be taken into account in efficiency loss calculation with increase in the operating temperature. It has been shown that the results of theoretical modeling the temperature dependences for the short-circuit current density, open-circuit voltage and photoconversion efficiency are in good agreement with the experimental data obtained using the sunlight simulator. These results could be used to develop methods for investigation of temperature dependences of solar cell characteristics by using various sunlight simulators.
\end{abstract}

Keywords: silicon solar cell, photoconversion efficiency, sunlight simulator.

Manuscript received 02.03.15; revised version received 05.06.15; accepted for publication 03.09.15; published online 30.09.15.

\section{Introduction}

Characterization of the solar cell (SC) parameters by using AM0 and AM1.5 sunlight simulators is developed rather good [1-6], however methods for measuring the temperature dependences of SC characteristics only partially take into account peculiarities of physical processes occurring in SC. With certain restrictions, temperature dependences of SC characteristics were described in monographs $[7,8]$. But there is no specific physical analysis for the case of incandescent lamps in standards [1-6] or in the monographs $[7,8]$. Our researches show that the short-circuit current temperature growth is substantially increased and the temperature fall of photocon- version efficiency is decreased in this case. This is caused by the shift of the spectral maximum to long-wave region with blackbody effective temperature decrease. The effect should be taken into account in measurements of temperature dependences of SC characteristics when using sunlight simulators. Since the SC operational temperature is almost always greater than $298 \mathrm{~K}\left(25^{\circ} \mathrm{C}\right)$, the temperature coefficient of photoconversion efficiency for sunlight simulator can significantly differ from that for natural conditions. The reason of this difference lies in the red shift for most of incandescent lamp spectra relatively to the Sun spectrum.

This difference is particularly essential for SCs manufactured using high-quality silicon exhibiting high 
values of bulk lifetimes and diffusion lengths. As far as the current growing coefficient of such SC, especially for natural terrestrial conditions, is significantly lower than that under sunlight simulator, the photoconversion efficiency for simulator will decrease to lower values than for natural conditions.

An experimental study of the temperature dependences for short-circuit current, open-circuit voltage, photoconversion efficiency and temperature coefficient of photoconvertion efficiency for silicon SCs with high volume lifetimes under sunlight simulator utilizing incandescent lamps have been presented in this paper. A comparison of the experimental results with the theory that takes into account formation of the opencircuit voltage in structures with high bulk lifetimes has been performed. It has been shown that the agreement between experimental data and theory is quite well. In addition, the temperature dependences of SC characteristics have been calculated theoretically for extraterrestrial conditions (AM0). It has been ascertained that these dependences significantly differ from those for the sunlight simulator.

These results can be used to develop methodologies for studying the temperature dependences of SC characteristics using various solar simulators.

\section{The experimental results}

The temperature dependences of short-circuit current density $J_{S C}$, open-circuit voltage $V_{O C}$ and efficiency $\eta$ were investigated using five samples. The first sample was heterojunction with a thin intrinsic layer (HIT) SC, the other four ones were silicon SCs with $p-n$ junctions, three of them were almost identical. Measurements were conducted for one HIT SC and one silicon SC with diffused $p-n$ junction by using AM1.5 conditions, as well as for two silicon SCs with diffused $p-n$ junctions by using AM0 conditions. Diffused $p-n$ junction silicon SC with a small bulk lifetime was measured under AM0 conditions for comparison.
We emphasize that AM0 and AM1.5 simulation by using incandescent lamps means normalization of maximum short-circuit density to 53.65 and $43.18 \mathrm{~mA} / \mathrm{cm}^{2}$, respectively, for $T=25^{\circ} \mathrm{C}$.

Measurements were based on the international and national standards [1-6]. Relative error was less than $\pm 0.1 \%$ for the short-circuit current, $\pm 0.05 \%$ for the opencircuit voltage and $\pm 1 \%$ for the photoconversion efficiency.

The initial silicon parameters and main SC characteristics for AM1.5 and AM0 conditions are shown in Table.

All examined SCs were manufactured from $n$-type silicon. Samples 1-4 had a great bulk lifetime. These samples satisfy inequality $L>d$, where $L$ is the diffusion length for non-equilibrium electron-hole pairs. The first sample (the so-called HIT SC) has the smallest surface recombination velocity equal to $40 \mathrm{~cm} / \mathrm{s}$. HIT SC manufacturing includes cleaning the crystalline silicon substrate, texturing the surfaces (formation of the pyramidal surface relief), deposition of undoped hydrogenated amorphous silicon $\alpha$-Si:H layer with the approximately $10-\mathrm{nm}$ thickness on both surfaces. The front anisotype $n$-cSi $/\left(p^{+}\right) \alpha-\mathrm{Si}: \mathrm{H}$ heterojunction and rear isotype $n$-cSi $/\left(n^{+}\right) \alpha-\mathrm{Si}: \mathrm{H}$ heterojunction were formed next. To reduce the series resistance on both surfaces, the transparent conductive layers were deposited from the mixture of indium and tin oxides (ITO), which followed by low-temperature annealing. Finally, the grid contact was deposited on the front surface, and the solid contact was deposited on the rear surface.

Samples 2-4 were manufactured using the same technology, and they had diffused $p-n$ junctions. The $p$ type region of $p-n$ junctions with 0.6 to $0.8-\mu \mathrm{m}$ depth was formed near the front surface by the boron diffusion. The isotype $n-n^{+}$junction was formed near the rear surface by using phosphorus diffusion. To reduce the surface recombination rate and thermal oxidation, circumferential etching of $p-n$ junction was done. However, as Table shows, the surface recombination

\begin{tabular}{|c|c|c|c|c|c|c|c|c|c|c|}
\hline $\begin{array}{c}\text { Sample } \\
\#\end{array}$ & $\begin{array}{c}\text { AM } \\
1.5\end{array}$ & AM0 & $\begin{array}{c}N_{d}, \\
\mathrm{~cm}^{-3}\end{array}$ & $\begin{array}{c}d, \\
\mu \mathrm{m}\end{array}$ & $\begin{array}{c}\tau_{b}, \\
\mathrm{~ms}\end{array}$ & $\begin{array}{c}S, \\
\mathrm{~cm} / \mathrm{s}\end{array}$ & $\begin{array}{c}J_{S C,} \\
\mathrm{~mA} / \mathrm{cm}^{2}\end{array}$ & $\begin{array}{c}V_{O C,} \\
\mathrm{~V}\end{array}$ & $\begin{array}{c}R_{s}^{*}, \\
\mathrm{Ohm} \cdot \mathrm{cm}^{2}\end{array}$ & $\begin{array}{c}\eta, \\
\%\end{array}$ \\
\hline 1 & + & & $1.6 \cdot 10^{15}$ & 300 & 1.4 & 40 & 29.9 & 0.675 & 5.7 & 12.3 \\
\hline 2 & + & & $3.1 \cdot 10^{15}$ & 380 & 0.4 & 200 & 30.6 & 0.631 & 0.15 & 16 \\
\hline 3 & & + & $3.1 \cdot 10^{15}$ & 380 & 0.4 & 380 & 40.5 & 0.630 & 1.1 & 14.5 \\
\hline 4 & & + & $3.1 \cdot 10^{15}$ & 380 & 0.4 & 330 & 40.4 & 0.631 & 1.18 & 14.3 \\
\hline 5 & & + & $3.1 \cdot 10^{15}$ & 380 & 0.018 & 3500 & 26.7 & 0.556 & 1.1 & 8.27 \\
\hline
\end{tabular}

Note. Here, $N_{d}$ is the doping concentration, $d$ - thickness of the sample, $\tau_{b}$ - bulk lifetime, $S$ - surface recombination velocity, $R_{s}^{*}$ - product of series resistance on the sample area. 
velocity $S$ for SCs with diffused $p-n$ junctions is not lowered to the values close to $40 \mathrm{~cm} / \mathrm{s}$ (obtained for HIT SCs).

The fifth sample was also made on $n$-type silicon with the high bulk lifetime, but during thermal oxidation temperature was higher. So, the bulk lifetime fell significantly.

\section{Modeling the temperature dependences of the basic silicon solar cell characteristics when using the simulated sunlight}

At first, let us to analyze the temperature dependence of short-circuit current density $J_{S C}$. In general, we can write

$J_{S C}(T)=q\left(1-r_{L}\right) \int_{\lambda_{0}}^{\lambda_{m}(T)} q_{c}(\lambda, T) I(\lambda) d \lambda$,

where $q$ is the elementary charge, $r_{L}$ is the average photocurrent loss factor associated with reflection, incomplete light absorption and presence of a contact grid on the front surface, $\lambda_{m}(T)=1.24 / E_{g}(T)-$ limit of the photoelectric effect (absorption edge), $E_{g}(T)$ - bandgap, $\lambda_{0}-\mathrm{SC}$ blue limit, $q_{c}(\lambda, T)-$ photocurrent collection coefficient, $I(\lambda)$ - spectral dependence of the rate of electron-hole pair generation in semiconductor that depends on the sunlight or sunlight simulator characteristics.

For black or grey body as a radiation source, according to Planck's formula

$$
J_{S C}(T)=q\left(1-r_{L}\right) B_{0} \int_{\lambda_{0}}^{\lambda_{m}(T)} \frac{q_{c}(\lambda, T) d \lambda}{\lambda^{4}\left(\exp \left(\frac{h c}{k T_{r} \lambda}\right)-1\right)},
$$

where $B_{0}$ is a constant, $h$ - Planck's constant, $c-$ light velocity, $k$ - Boltzmann constant, $T_{r}-$ black body temperature.

$$
B_{0}=2 \pi c\left(r_{s} / D_{s}\right)^{2} \text { for AM0 conditions [9]. Here, }
$$

$r_{s}$ is the radius of Sun, $D_{S}-$ distance from Earth to Sun, $T_{S}=5800 \mathrm{~K}, T=298 \mathrm{~K}$. For the sunlight simulator with an incandescent lamp, the photocurrent equality for the photocurrent generated by Sun and photocurrent generated by the lamp can be written as

$$
\begin{aligned}
& 2 \pi c\left(\frac{r_{s}}{D_{s}}\right)^{2} \cdot \int_{\lambda_{0}}^{\lambda_{m}(298 K)} \frac{q_{c}(\lambda, T) d \lambda}{\lambda^{4}\left(\exp \left(\frac{h c}{5800 k \lambda}\right)-1\right)}= \\
& =f\left(R_{L}\right) \cdot \int_{\lambda_{0}}^{\lambda_{m}(298 K)} \frac{q_{c}(\lambda, T) d \lambda}{\lambda^{4}\left(\exp \left(\frac{h c}{k T_{L} \lambda}\right)-1\right)},
\end{aligned}
$$

where $T_{L}$ is the temperature of the incandescent lamp, $f\left(R_{L}\right)$ - function that describes the intensity of simulated radiation at the distance $R_{L}$ to SC.
The short-circuit current density $J_{S C}$ temperature growth for AM0 conditions can be described by the factor

$$
\begin{aligned}
& F(T)=\int_{\lambda_{0}}^{\lambda_{m}(T)} \frac{q_{c}(\lambda, T) d \lambda}{\lambda^{4}\left(\exp \left(\frac{h c}{k T_{r} \lambda}\right)-1\right)^{-1}} \times \\
& \times\left(\int_{\lambda_{0}}^{\lambda_{m}(298 K)} \frac{q_{c}(\lambda, T) d \lambda}{\lambda^{4}\left(\exp \left(\frac{h c}{k T_{r} \lambda}\right)-1\right)}\right)
\end{aligned}
$$

For $L>>d, q_{c}(\lambda) \approx 1$ and (4) can be simplified to

$$
\begin{gathered}
F(T)=\int_{\lambda_{0}}^{\lambda_{m}(T)} \frac{d \lambda}{\lambda^{4}\left(\exp \left(\frac{h c}{k T_{r} \lambda}\right)-1\right)^{-1}} \times \\
\times\left(\int_{\lambda_{0}}^{\lambda_{m}(298 K)} \frac{d \lambda}{\lambda^{4}\left(\exp \left(\frac{h c}{k T_{r} \lambda}\right)-1\right)}\right)^{-1} .
\end{gathered}
$$

In this case, $J_{S C}(T)=J_{S C}\left(25^{\circ} \mathrm{C}\right) F(T)$.

Fig. 1 shows the theoretical spectral dependence of radiation intensity normalized to the maximum value, and Fig. 2 shows $F(T)$ dependences for silicon SC plotted using the expression (5). The figures correspond to different illuminations. The AM1.5 curve of Fig. 2 was calculated by the numerical methods using the standard procedure $[10,11]$. Fig. 1 shows that the lower is the radiation temperature, the larger is the red shift of spectral distribution. Since $\lambda_{m}$ increases with ambient temperature growth, $F$ increases with $T_{r}$ decrease.

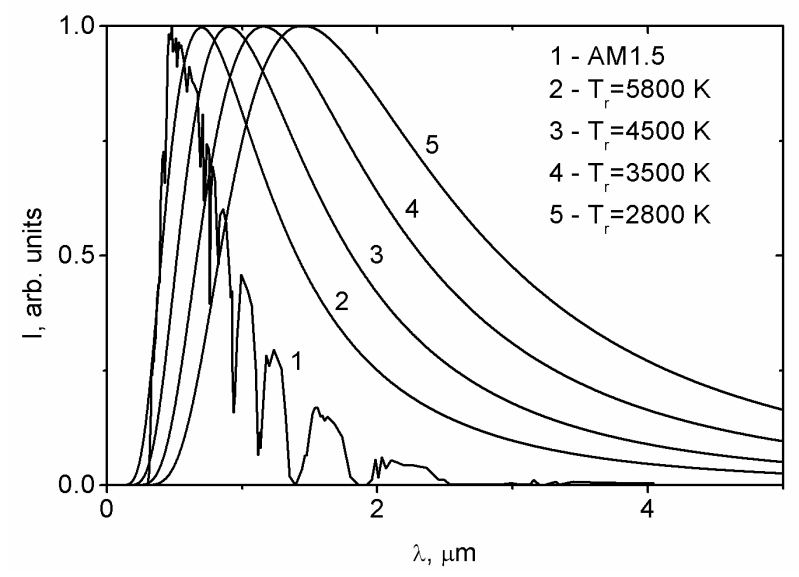

Fig. 1. Solar spectral irradiance distributions. 


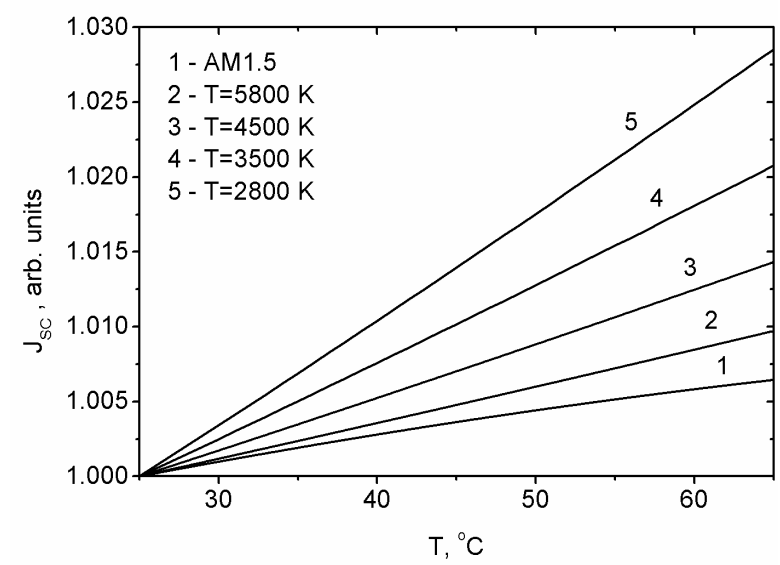

Fig. 2. Silicon SCs short-circuit current temperature dependences for different spectral irradiance distributions.

Comparing the $F$ values for $T_{r}=5800 \mathrm{~K}$ (temperature of solar radiation) and $T_{r}=2800 \mathrm{~K}$ (incandescent lamp temperature), one can see that for the SC temperature growth from 25 to $65{ }^{\circ} \mathrm{C}$, the short-circuit current $J_{S C}$ increases by $3 \%$ for $T_{r}=2800 \mathrm{~K}$ and $1 \%$ for $T_{r}=$ $5800 \mathrm{~K}$. Thus, the use of solar simulators with incandescent lamps leads to a significant increase in the rate of short-circuit current temperature growth. This effect must be considered in the study of temperature dependences of the key SC characteristics first of all for the short-circuit current and photoconversion efficiency.

As stated above, $L>d$ condition is valid for the samples 1-4. Therefore, modeling temperature dependences for the open-circuit voltage $V_{O C}$ and efficiency $\eta$ can be done using the theory proposed in [12], which takes into account the possibility of nonlinear excitation $\left(\Delta p \geq N_{d}\right.$, when the excess concentration of electron-hole pairs takes place in the base).

According to [12], in the case of $n$-type base the open-circuit voltage $V_{O C}$ can be found using the expression:

$V_{O C} \cong \frac{k T}{q} \ln \left(\frac{\Delta p N_{d}}{n_{i}^{2}(T)}\right)+\frac{k T}{q} \ln \left(1+\frac{\Delta p}{N_{d}}\right)$,

where $T$ is SC temperature, $n_{i}(T)$ is the intrinsic charge carriers concentration in silicon.

The excessive concentration of electron-hole pairs $\Delta p$ can be found from the generation and recombination balance equation [12]

$$
J_{S C} / q=\left[\frac{d}{\tau_{b}}+S\right] \Delta p
$$

Here, $J_{S C}$ is the short-circuit current density, $\tau_{b}=\left(\tau_{S R}^{-1}+A\left(N_{d}+\Delta p\right)+\tau_{\text {Auger }}^{-1}\right)^{-1}-$ bulk lifetime, $\tau_{S R}-$ Shockley-Read-Hall lifetime, $A \approx 6.3 \cdot 10^{-15} \mathrm{~cm}^{3} / \mathrm{s}$ [13] radiative recombination coefficient in silicon, $\tau_{\text {Auger }}$ interband Auger recombination lifetime:

$\tau_{\text {Auger }}^{-1}=C_{p}\left(N_{d}+\Delta p\right) \Delta p+C_{n}\left(N_{d}+\Delta p\right)^{2}$,

where

$$
C_{n}=\left(2.8 \cdot 10^{-31}+\frac{2.5 \cdot 10^{-22}}{\left(N_{d}+\Delta p\right)^{0.5}}\right) \mathrm{cm}^{6} / \mathrm{s},
$$

$C_{p}=10^{-31} \mathrm{~cm}^{6} / \mathrm{s} \quad[14,15] ; S=S_{0}+S_{d}$ is the sum of surface recombinations on the front $S_{0}$ and rear $S_{d}$ surfaces. The expression (6) is a quadratic equation by $\Delta p$. It can be solved as

$\Delta p=-\frac{N_{d}}{2}+\sqrt{\frac{N_{d}^{2}}{4}+n_{i}^{2} \exp \left(\frac{q V_{O C}}{k T}\right)}$.

To get $\Delta p(V)$ function, we can replace $V_{O C}$ in (9) by the forward bias $V$ :

$\Delta p(V)=-\frac{N_{d}}{2}+\sqrt{\frac{N_{d}^{2}}{4}+n_{i}^{2} \exp \left(\frac{q V}{k T}\right)}$.

SC photocurrent density in the $V<V_{O C}$ range is

$J(V)=J_{S C}-J_{\text {rec }}(V)$,

where

$J_{\text {rec }}(V)=q\left(\frac{d}{\tau_{b}}+S\right) \Delta p(V)$.

Eq. (12) is the expression for SC current-voltage characteristic in quadratures, which takes into account the complex bulk lifetime $\tau_{b}$ dependence on the excess concentration $\Delta p(V)$.

For the maximum power take-off condition $d(V J(V)) / d V=0$, the photovoltage $V_{m}$ and current density $J_{m}$ can be found. The photoconversion efficiency $\eta$ for silicon $\mathrm{SC}$ with a unit area and series resistance $R_{s}$ can be written as

$\eta=\frac{J_{m} V_{m}}{P_{S}}\left(1-\frac{J_{m} R_{s}}{V_{m}}\right)$,

where $P_{s}$ is the power of incident solar radiation.

For the $L<d / 2$ case (sample No. 5), the general expression (1) must be used to calculate the short-circuit current and its temperature increase. In this case, the expression for photocurrent collection has the form

$q(\lambda, T)=\frac{\alpha(\lambda, T) L(T)}{1+\alpha(\lambda, T) L(T)}$, 
where $\alpha(\lambda, T)$ is the light absorption coefficient for semiconductor.

In the case of small bulk lifetimes $\tau_{b}$, the excess concentration $\Delta p$ is low, as compared to the concentration of majority carriers, so the open-circuit voltage $V_{O C}$ can be found using the formula

$V_{O C} \cong \frac{k T}{q} \ln \left(\frac{J_{S C}(T) N_{d}}{q\left(S+\frac{D(T)}{L(T)}\right) n_{i}^{2}(T)}\right)$

where $D(T)$ is the diffusion coefficient for holes.

The photoconversion efficiency $\eta$ for the unit area $\mathrm{SC}$ under AM1.5 illumination is

$\eta \cong \frac{J_{S C} V_{O C}}{0.1}\left(1-\frac{k T}{q V_{O C}}\right)\left(1-\frac{k T \ln \left(q V_{O C} / k T\right)}{q V_{O C}}\right) \times$

$\times\left(1-\frac{R_{s} J_{S C}}{V_{O C}}\right)$

It should be noted that the temperature dependences of photoconversion efficiency $\eta$ are primarily determined by the open-circuit voltage $V_{O C}$ and fill factor $F F$ of current-voltage characteristic $[7,8,16,17]$. $V_{O C}$ and $F F$ decrease is mainly caused by the intrinsic concentration $n_{i}$ exponential growth with temperature $\left(n_{i} \propto \exp \left[-q E_{g} / 2 k T\right]\right)$. The larger the semiconductor band gap $E_{g}$, the less the temperature decrease of the coefficient $\beta$ for the photoconversion efficiency $\eta$.

To calculate the temperature dependence for the open-circuit voltage $V_{O C}$, we must take into account $n_{i}(T)$ dependence as well as temperature dependences for the Shockley-Read-Hall lifetime $\tau_{\mathrm{SR}}$, radiative recombination lifetime, Auger recombination lifetime and surface recombination velocity. Finally, the shortcircuit current density $J_{S C}$ temperature dependence should be considered. Since these dependences are mostly power laws (opposed to the exponential $n_{i}(T)$ dependence), they will in general give small corrections to $V_{O C}(T)$ dependence as compared to $n_{i}(T)$ contribution.

Let us analyze these dependences.

For the arbitrary excitation, $\tau_{S R}$ has the following form where $C_{p}(T)=V_{p} \sigma_{p}(T) \quad$ and $\quad C_{n}(T)=V_{n} \sigma_{n}(T)$ are electrons and holes capture coefficients inherent to the recombination center, $E_{i}$ is the energy position and $N_{t}$ concentration of recombination centers. As shown in [18], the holes capture cross section $\sigma_{p}(T)$ depends on temperature as $T^{-2}$ for neutral recombination centers and as $T^{-1}$ up to $T^{-3}$ for the attractive centers. Therefore, at low excitation levels, taking into account the thermal velocity temperature dependence, we obtained $\tau_{\mathrm{SR}} \propto T^{3 / 2}$ for $\sigma_{p}(T) \propto T^{-2} \quad$ [8]. For the repulsive center $\sigma_{p}(T) \propto \exp \left(-\left(T_{0} / T\right)^{1 / 3}\right)$ [18]. In this case $\tau_{\mathrm{SR}}(T)$ decrease with temperature increase. It should be noted that in this case the $\sigma_{p}(T)$ dependence is significantly weaker than the $n_{i}(T)$ dependence.

$\tau_{\mathrm{SR}}(T)$ variation with the temperature increase from 25 up to $65^{\circ} \mathrm{C}$, as compared to $n_{i}(T)$ variation, is estimated as not exceeding $0.5 \%$ for $\mathrm{Si}$ under low excitation. $\tau_{\mathrm{SR}}(T)$ dependence is weak compared to $T^{3 / 2}$ or even absent for sufficiently high excitation $\left(\Delta p \geq N_{d}\right)$. The situation with $\tau_{\text {Auger }}$ temperature dependences is similar [18]. $\tau_{r}$ exceeds $10^{-2} \mathrm{~s}$ for the doping of about $10^{15} \mathrm{~cm}^{-3}$, so the radiative recombination could be neglected relative to ShockleyRead-Hall recombination.

The temperature dependence of the surface recombination velocity $S$ in silicon $\mathrm{SC}$ has not been investigated in detail. However, it is expected that $S(T)$ dependence and temperature dependence of the bulk lifetime obey power laws. Therefore, in further calculations it can be assumed that $S(T)$ dependence may be neglected.

In silicon, with the doping level of $10^{15} \mathrm{~cm}^{-3} D$ depends on temperature approximately as $T^{-1.2}$.

So, the temperature dependences of short-circuit current density and the intrinsic carriers concentration have to be taken into account in the temperature dependences modeling for the key characteristics of the samples 1-4. For the sample 5, temperature dependences of the bulk lifetime and minority carrier diffusion coefficient have to be considered.

Figs. 3 to 5 present experimental data for shortcircuit current density $J_{S C}$, open-circuit voltage $V_{O C}$ and efficiency $\eta$, respectively. The numbers of curves correspond to those of the samples listed in Table. Experimental parameters are compared with the theoretically predicted ones. Calculations for the samples 1-4 were made using the expressions (6)-(13).

$$
\tau_{\mathrm{SR}} \equiv \frac{C_{p}(T)\left(p_{0}+\Delta p+n_{i}(T) \exp \left(E_{i} / k T\right)\right)+C_{n}(T)\left(n_{0}+\Delta p+n_{i}(T) \exp \left(-E_{i} / k T\right)\right)}{C_{p}(T) C_{n}(T) N_{t}\left(n_{0}+\Delta p\right)},
$$




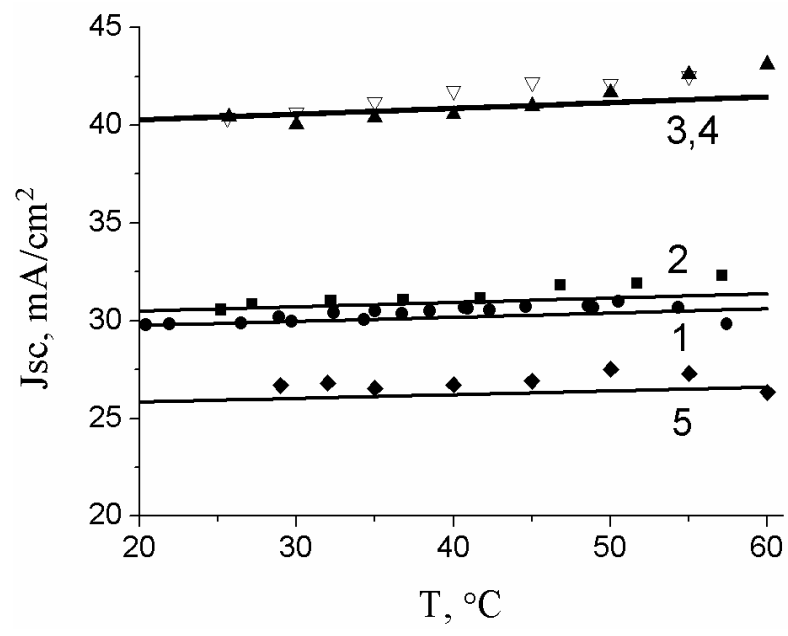

Fig. 3. Silicon SCs short-circuit current temperature dependences for various samples. Points for experiment, lines for the theory. The numbers of curves correspond to those of the samples listed in Table.

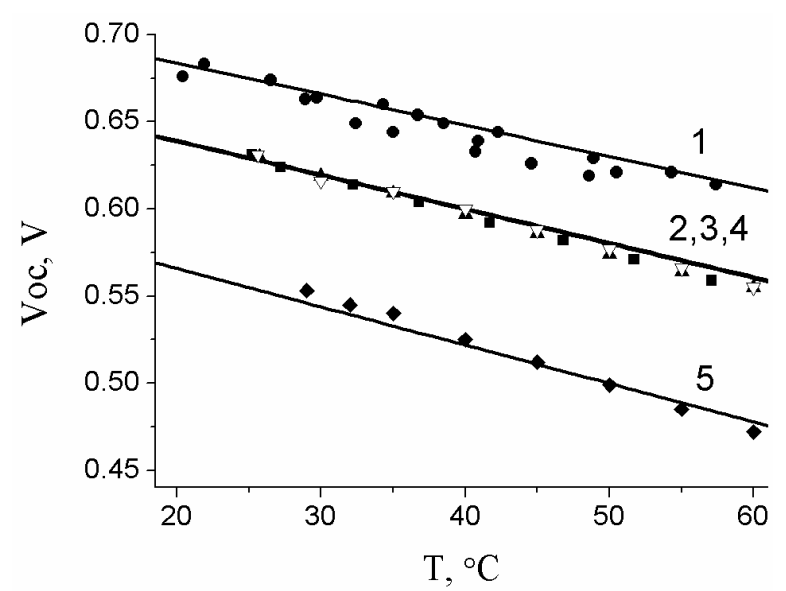

Fig. 4. Silicon SCs open-circuit voltage temperature dependences for various samples. Points for experiment, lines for the theory. The numbers of curves correspond to those of the samples listed in Table.

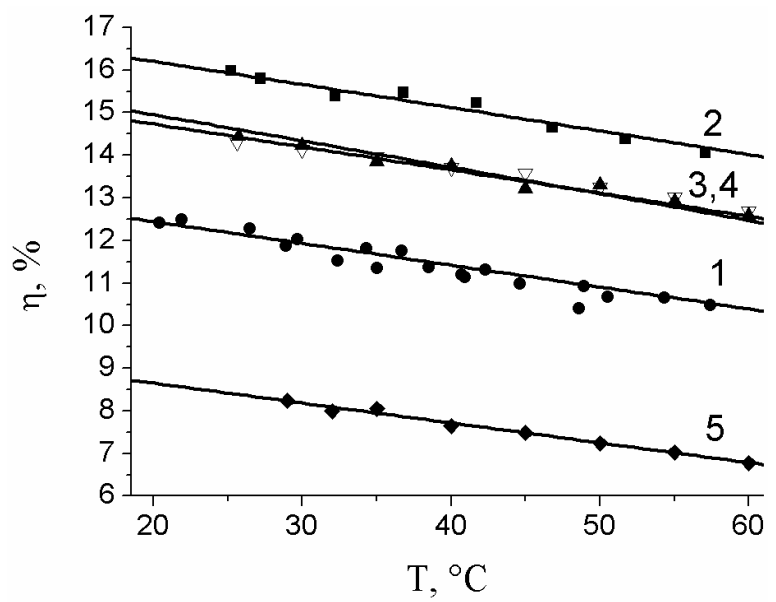

Fig. 5. Silicon SCs photoconversion efficiency temperature dependences for various samples. Points for experiment, lines for the theory. The numbers of curves correspond to those of the samples listed in Table.
Formulas (14)-(17) were used for the sample 5. A good agreement between the experimental and calculated parameters is obvious.

Fig. 6 shows the results for the average temperature fall coefficient $\bar{\beta}(T)$ of photoconversion efficiency. The numbers of curves correspond to those of the samples listed in Table. To calculate $\bar{\beta}(T)$ dependence (in percent), we used the expression

$$
\bar{\beta}(T)=\frac{\eta\left(T_{0}\right)-\eta(T)}{\left(\eta\left(T_{0}\right)+\eta(T)\right)\left(T-T_{0}\right)} 200,
$$

where $T_{0}$ is the starting temperature, which in this case is equal to $298 \mathrm{~K}\left(25^{\circ} \mathrm{C}\right)$.

Fig. 6 shows that the $\bar{\beta}(T)$ values vary quite widely. The samples 2 to 4 have the minimal $\bar{\beta}(T)$ values (near $0.35 \ldots 0.4 \% / K) . \bar{\beta}(T)$ values for samples 1 and 5 are higher. The sample 5 has the greatest $\bar{\beta}(T)$ maximum value (more than $0.5 \% / \mathrm{K}$ ). $\bar{\beta}(T)$ values depend on the bulk and surface recombination total velocity. This velocity is lower, the less is the $\bar{\beta}(T)$ value. On the other hand, the large series resistance $R_{S}$ causes $\bar{\beta}(T)$ increase. The fill factor $F F$ also strongly depends on $R_{S}$. In our samples, the serial resistance first of all is determined by the parameters of heavily doped emitter. The higher doped emitter and the greater the thickness, the lower series resistance. However, interband Auger recombination is increased in this case, so the short-circuit current decreases. The series resistance value for the HIT elements is also determined by ITO film parameters. The higher film thickness, the lower series resistance. However, the increase in ITO film thickness increases light absorption in this film. This effect also leads to a decrease in the short-circuit current. Thereby the thickness of the emitter and thickness of the ITO film have to be optimized.

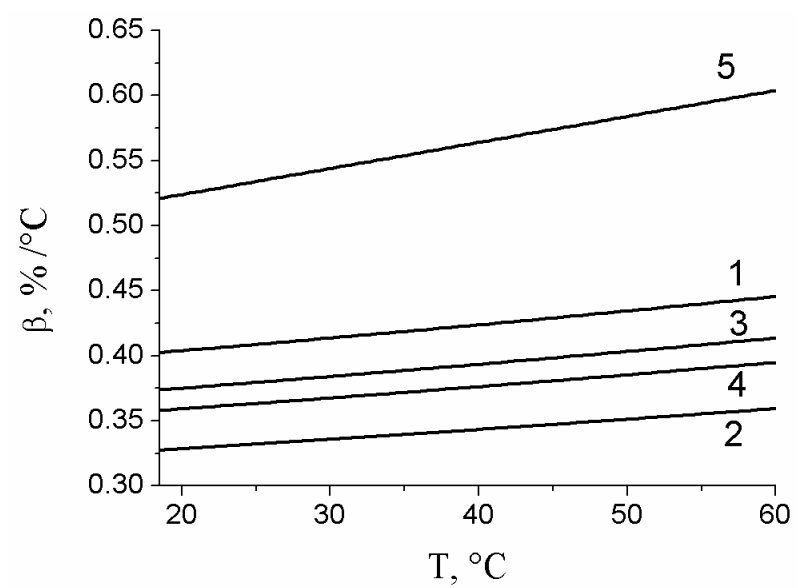

Fig. 6. Silicon SCs averaged photoconversion efficiency fall coefficient temperature dependences for various samples. The numbers of curves correspond to those of the samples listed in Table.

\section{(C) 2015, V. Lashkaryov Institute of Semiconductor Physics, National Academy of Sciences of Ukraine}




\section{Results and discussion}

Now we carry out an analysis of the incandescent light simulator features influence on the temperature dependence of the key silicon SC characteristics. For the short-circuit current, this influence was actually analyzed at the beginning. It was shown that the use of such simulators significantly accelerate growth of the short-circuit current. In natural conditions (inter alia for AM1.5 conditions), short-circuit current is much less increased as compared to the simulated illumination.

Temperature dependences of the open-circuit voltage $V_{O C}$ and photoconversion efficiency $\eta$ were calculated both for black-body temperature $2800 \mathrm{~K}$ (corresponding to the incandescent lamp) and $5800 \mathrm{~K}$ (corresponding to AM0 conditions) to show the difference of these cases for the sample 4 . $V_{O C}(T)$ dependences for AM0 and simulated spectra practically coincide, but there is a little difference for $\eta(T)$ (see Fig. 7). Fig. 8 shows more significant difference for the photoconversion efficiency temperature coefficients $\bar{\beta}(T)$.

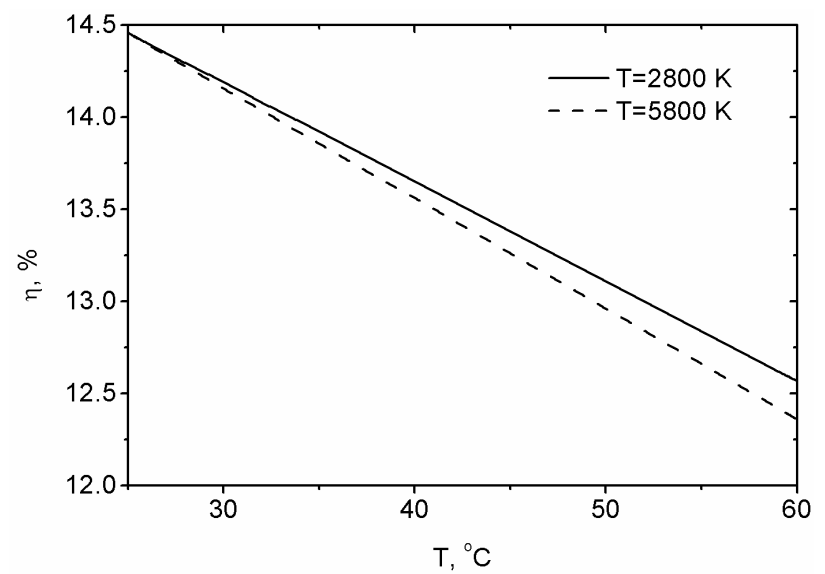

Fig. 7. Theoretical photoconversion efficiency for the sample 4 vs. temperature. The black body radiation temperatures of 2800 and $5800 \mathrm{~K}$ were applied for illumination.

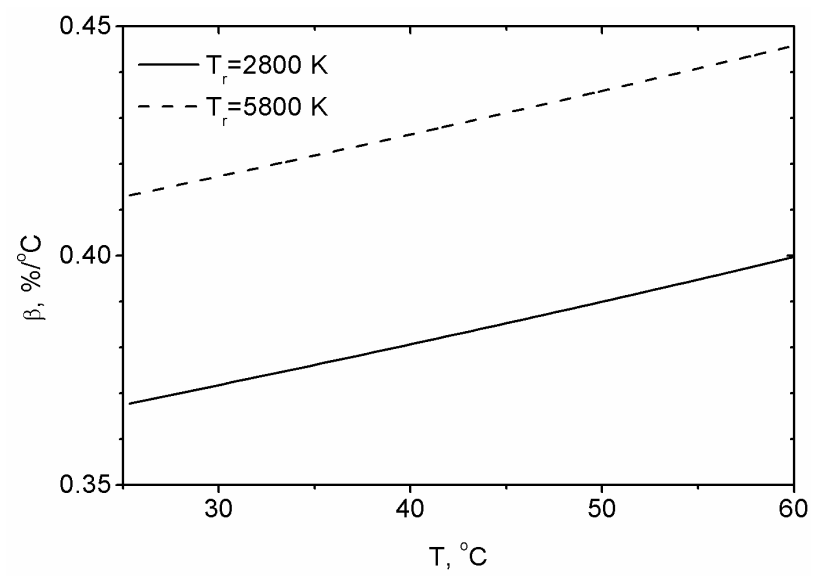

Fig. 8. Theoretical photoconversion efficiency fall coefficient for the sample 4 vs. temperature. The black body radiation temperatures of 2800 and $5800 \mathrm{~K}$ were applied for illumination.
As seen from Fig. 8, $\bar{\beta}(T)$ for simulator is lower than for AM0. It is caused by high short-circuit current $J_{S C}$ growth under simulated illumination. The effect of this growth compensates partly contribution of the intrinsic concentration $n_{i}$ temperature dependence. So, the photoconversion efficiency temperature coefficient $\bar{\beta}(T)$ is virtually decreased. But for the actual SC operation conditions, this strong short-circuit current growth is absent, and therefore $\bar{\beta}(T)$ value is higher. This effect should be taken into account in calculations of the real photoconversion efficiency drop with the temperature increase.

\section{Conclusion}

Temperature dependences for such SC parameters as short-circuit current density, open-circuit voltage and photoconversion efficiency are investigated for different samples under simulated solar illumination. It is shown that the use of incandescent lamps leads to a significant increase in short-circuit current growth.

Temperature dependences of these SC parameters were calculated for both high diffusion length (relative to the thickness of the sample) with high excitation and the opposite case of low diffusion length with low excitation. A good agreement between the theoretical calculations and experimental results has been achieved.

It has been found that, in the case of sunlight simulators equipped with incandescent lamps, the increase in short-circuit current growth with temperature leads to reduction in the photoconversion efficiency temperature fall coefficient. De facto, in actual operation conditions this reduction is absent. This difference has to be taken into account in the SC power temperature losses estimation.

Our approach, in principle, allows to calculate the temperature dependences of the basic SC parameters for different types of solar simulators.

\section{References}

1. A.P. Gorban, V.P. Kostylyov, A.V. Sachenko, About increasing accuracy of determining photoenergetic parameters of photoconvertors on sunlight simulators uncorrected in accord with the spectrum // Netraditsionnye istochniki, peredaiuschie sistemy $i$ preobrazovateli energii. Sbornik nauchnykh statei. vol. 1. Kharkov, p. 3334, 1997 (in Russian).

2. PV Power PV Standarts // http://www.senes.bas.bg/pv_standarts.htm

3. GOST 28976-91. Photoelectric devices made of crystalline silicon. Method for correction by temperature and irradiance of the results of measuring the current-voltage characteristics (in Russian). 
4. M.I. Klyuy, V.P. Kostylyov, A.V. Makarov, V.V. Chernenko, Metrologic aspects of testing photoelectric covertors of solar energy // Skladni systemy i protsesy, 1, p. 42-50 2007 (in Ukrainian).

5. V.P. Kostylyov, V.V. Chernenko, S.P. Andros, L.A. Nazarenko, Metrology and standardization of phototechnical tests for photoelectric covertors of solar energy and photoelectric modules // Modern problems of light engineering and electrical energetics: Materials of IV Intern. Sci.-techn. Conf. Kharkiv, April 13-14, 2011. Kharkiv National Academy of Municipal Economy, p. 164-165 (in Ukrainian).

6. A.P. Gorban, V.P. Kostylyov, V.V. Chernenko, S.P. Andros, L.A. Nazarenko, Modern problems of simulation of solar emission for testing photoelectric covertors of solar energy // Modern problems of light engineering: Materials of III Intern. Sci.-techn. Conf. Kharkiv, April 22-23, 2009 (in Ukrainian).

7. A.M. Vasilev, A.P. Landsman, Semiconductor Photoconvertors. Soviet Radio Publishers, Moscow, 1971 (in Russian).

8. A.L. Fahrenbruch, R.H. Bube, Fundamentals of Solar Cells. Photovoltaic Solar Energy Conversion. New York, 1983.

9. G.E. Pikus, Fundamentals of the Theory of Semiconductor Devices. Nauka, Moscow, 1965 (in Russian).

10. ASTM G173-03(2012), Standard Tables for Reference Solar Spectral Irradiances: Direct Normal and Hemispherical on $37^{\circ}$ Tilted Surface, ASTM International, West Conshohocken, PA, 2012 // www.astm.org
11. ISO 9845-1:1992 Solar energy - Reference solar spectral irradiance at the ground at different receiving conditions - Part 1: Direct normal and hemispherical solar irradiance for air mass 1,5. http://www.iso.org

12. A.V. Sachenko, A.I. Shkrebtii, R.M. Korkishko, V.P. Kostylyov, N.R. Kulish, I.O. Sokolovskyi, Features of photoconversion in highly efficient silicon solar cells // Semiconductors, 49(2), p. 264269 (2015).

13. A.P. Gorban, A.V. Sachenko, V.P. Kostylyov, N.A. Prima, Effect of excitons on photoconversion efficiency in the $\mathrm{p}^{+}-\mathrm{n}-\mathrm{n}^{+}-$and $\mathrm{n}^{+}-\mathrm{p}-\mathrm{p}^{+}$-structures based on single-crystalline silicon // Semiconductor Physics, Quantum Electronics and Optoelectronics, 3(3), p. 322 (2000).

14. A.V. Sachenko, A.P. Gorban, V.P. Kostylyov, I.O. Sokolovsky, The radiative recombination coefficient and the internal quantum yield of electroluminescence in silicon // Semiconductors, 40(8), p. 884-889 (2006).

15. A. Hangleiter, R. Häcker, Enhancement of band-toband Auger recombination by electron-hole correlations // Phys. Rev. Lett. 65(2), p. 215-218 (1990).

16. http://solar-front.livejournal.com/11644.html

17. E. Skoplaki, J.A. Palyvos, On the temperature dependence of photovoltaic module electrical performance: A review of efficiency/power correlations // Solar Energy, 83, p. 614-624 (2009).

18. V.N. Abakumov, V.I. Perel, I.N. Yassievich, Nonradiative Recombination in Semiconductors. Publ. House "Petersburg Institute of Nuclear Physics by name B.P. Konstantinov of RAN", S-Petersburg, 1997 (in Russian). 